\title{
Predicting fever in neutropenia with safety-relevant events in children undergoing chemotherapy for cancer: The prospective multicenter SPOG 2015 FN Definition Study
}

\author{
Luana Lavieri $^{1}$ | Christa Koenig ${ }^{1}$ () | Nicole Bodmer ${ }^{2}$ | Philipp K. A. Agyeman ${ }^{3}$ | \\ Katrin Scheinemann ${ }^{4,5,6} \quad$ | Marc Ansari ${ }^{7,8} \quad$ | Jochen Roessler ${ }^{1}$ (ㅇ \\ Roland A. Ammann ${ }^{1,9}$ (1) \\ ${ }_{1}^{1}$ Pediatric Hematology/Oncology, Department of Pediatrics, Inselspital, Bern University Hospital, University of Bern, Bern, Switzerland \\ ${ }^{2}$ Pediatric Oncology, University Children's Hospital of Zürich, University of Zürich, Zürich, Switzerland \\ ${ }^{3}$ Department of Pediatrics, Inselspital, Bern University Hospital, University of Bern, Bern, Switzerland \\ ${ }^{4}$ Pediatric Hematology/Oncology, Department of Pediatrics, Kantonsspital Aarau, Aarau, Switzerland \\ 5 University of Basel, Basel, Switzerland \\ ${ }^{6}$ Department of Pediatrics, McMaster Children's Hospital and McMaster University, Hamilton, Ontario, Canada \\ ${ }^{7}$ Pediatric Hematology/Oncology, Department of Women, Child and Adolescent, University Hospital of Geneva, Geneva, Switzerland \\ ${ }^{8}$ Department of Pediatrics, Gynecology, and Obstetrics, Cansearch Research Platform of Pediatric Oncology and Hematology, Faculty of Medicine, University of \\ Geneva, Geneva, Switzerland \\ ${ }^{9}$ Kinderaerzte KurWerk, Burgdorf, Switzerland
}

\section{Correspondence}

Roland A. Ammann, Pediatric Hematology/Oncology, Department of Pediatrics, Inselspital, Bern University Hospital, Murtenstrasse 10, CH-3010 Bern, Switzerland.

Email:roland.ammann@insel.ch

\section{Luana Lavieri and Christa Koenig shared first} authorship.

Funding information

Swiss Cancer League, Grant Number: KLS3645-02-2015; Berner Stiftung für krebskranke Kinder

Parts of the results reported here have been presented at the Annual SPOG Scientific Meeting, February 8, 2020, Lugano, Switzerland (no abstract published).

\begin{abstract}
Background: Fever in neutropenia (FN) remains a frequent complication in pediatric patients undergoing chemotherapy for cancer. Preventive strategies, like primary antibiotic prophylaxis, need to be evidence-based.

Procedure: Data on pediatric patients with any malignancy from the prospective multicenter SPOG 2015 FN Definition Study (NCT02324231) were analyzed. A score predicting the risk to develop FN with safety-relevant events (SRE; bacteremia, severe sepsis, intensive care unit admission, death) was developed using multivariate mixed Poisson regression. Its predictive performance was assessed by internal crossvalidation and compared with the performance of published rules.

Results: In 238 patients, 318 FN episodes were recorded, including 53 (17\%) with bacteremia and 68 (21\%) with SRE. The risk-prediction score used three variables: chemotherapy intensity, defined according to the expected duration of severe neu-
\end{abstract}


tropenia, time since diagnosis, and type of malignancy. Its cross-validated performance, assessed by the time needed to cover (TNC) one event, exceeded the performance of published rules. A clinically useful score threshold of $\geq 11$ resulted in $2.3 \%$ time at risk and 4.1 months TNC. Using external information on efficacy and timing of intermittent antibiotic prophylaxis, 4.3 months of prophylaxis were needed to prevent one FN with bacteremia, and 5.2 months to prevent one FN with SRE, using a threshold of $\geq 11$. Conclusions: This score, based on three routinely accessible characteristics, accurately identifies pediatric patients at risk to develop FN with SRE during chemotherapy. The score can help to design clinical decision rules on targeted primary antibiotic prophylaxis and corresponding efficacy studies.

KEYWORDS

antibiotic prophylaxis, febrile neutropenia, infections in immunocompromised hosts, neutropenia, pediatric oncology, risk-prediction score

\section{1 | INTRODUCTION}

Chemotherapy-induced neutropenia is a major risk factor for severe infections in patients with cancer. ${ }^{1}$ Despite improved medical management, fever in neutropenia (FN) continues to be associated with significant morbidity and mortality. ${ }^{2}$ Preventive strategies, like primary antibiotic prophylaxis, potentially reduce morbidity and mortality, but must be evidence- and risk-based. The efficacy of antibiotic prophylaxis, measured by the reduction of mortality and of $\mathrm{FN}$ with complications, must be weighted prudently against potential adverse events. $^{3}$

A current systematic review of randomized controlled trials on the efficacy of antibiotic prophylaxis in adult and pediatric patients with cancer and recipients of hematopoietic stem cell transplantation (HSCT) found antibiotic prophylaxis to significantly reduce bacteremia, but not overall mortality. ${ }^{4}$

In pediatric oncology, current clinical guidelines present conflicting and only weak recommendations for ${ }^{5}$ and against ${ }^{6}$ antibiotic prophylaxis during chemotherapy for selected patient groups. These discrepancies are explained by different methodologies in guideline development, but mainly reflect the paucity of clinical evidence on the efficacy of primary antibiotic prophylaxis beyond Pneumocystis jirovecii pneumonia prophylaxis.

Studies with a priori-defined, diagnosis-based risk groups only allow restricted conclusions. Power calculation for future studies remains difficult because of insufficient data on the potential efficacy of prophylaxis. ${ }^{7}$ For targeted primary antibiotic prophylaxis, better prediction of the risk to develop bacterial infections with complications during chemotherapy is needed. ${ }^{3}$

This analysis of data from pediatric patients undergoing chemotherapy for cancer aimed to analyze associations of clinical characteristics with the risk to develop FN with safety-relevant events (SRE; bacteremia, severe sepsis, intensive care admission, death) during chemotherapy; to derive a risk-prediction score for FN with SRE during chemotherapy; to estimate the score's performance including effect size; and to evaluate its performance compared with published scores or clinical decision rules.

\section{2 | METHODS}

\section{1 | Study design}

The prospective multicenter Swiss Paediatric Oncology Group (SPOG) 2015 FN Definition Study was opened for recruitment in six out of nine pediatric oncology centers in Switzerland from April 2016 to August 2018. Patients, if able to judge, and their legal guardians gave written informed consent (IC) before study entry. The study was conducted in accordance with the Declaration of Helsinki and the Swiss law, which refers to the current Good Clinical Practice guidelines. It had been registered at ClinicalTrials.gov (NCT02324231) and was approved by local ethics committees before patient recruitment. ${ }^{8}$ Data were collected and managed using REDCap electronic data capture tools. ${ }^{9}$

The SPOG 2015 FN Definition Study was designed to determine the safety of a higher $\left(39.0^{\circ} \mathrm{C}\right)$ versus lower $\left(38.5^{\circ} \mathrm{C}\right)$ fever limit using a nonblinded cluster-randomized controlled non-inferiority design. Patients, clustered via centers, were monthly randomized to one of the two fever limits. The $39.0^{\circ} \mathrm{C}$ fever limit has been found to be both safe and efficacious when compared to $38.5^{\circ} \mathrm{C}$; details have been published elsewhere. ${ }^{8}$ In this manuscript, an analysis of observational outcomes predefined in the protocol ${ }^{10}$ is reported.

\subsection{Patients and management of FN}

Patients treated with chemotherapy for cancer were consecutively screened. Inclusion criteria were age $\geq 12$ months and $<18$ years, diagnosis of any malignancy, treatment with myelosuppressive chemotherapy expected to last $\geq 2$ months, or greater than or equal to one cycle of myeloablative chemotherapy, followed by autologous HSCT, and written IC. Patients after allogeneic HSCT were excluded. ${ }^{8}$ After IC, 
patients were studied during the course of their chemotherapy. Information on clinical characteristics and FN, including the outcomes analyzed here, was collected each month.

Neutropenia was defined as an absolute neutrophil count $<0.5 \mathrm{G} / \mathrm{L}$, or $<1.0 \mathrm{G} / \mathrm{L}$ and expected to decline to $<0.5 \mathrm{G} / \mathrm{L}$ within 48 hours. ${ }^{11,12}$ Temperature was measured in the ear by infrared tympanic thermometry. $\mathrm{FN}$ was diagnosed in outpatients and inpatients when temperature reached the randomized fever limit $\left(38.5^{\circ} \mathrm{C}\right.$ or $\left.39.0^{\circ} \mathrm{C}\right)$, but diagnosis below this limit was allowed if clinically indicated. Diagnosis of FN implied emergency hospitalization, essential laboratory tests, and start of empirical intravenous broad-spectrum antimicrobial therapy. Coverage of Gram-positive cocci and Gram-negative bacteria was required, but a specific antianaerobic coverage was not. ${ }^{10}$ Each center chose antibiotics according to local resistance patterns. $^{8}$

In order to avoid a systematic bias, all patients from centers routinely applying primary antibiotic prophylaxis, beyond prophylaxis of Pneumocystis jirovecii pneumonia, in predefined patient groups were excluded from this analysis.

\section{3 | Outcomes}

Restarting antibiotics within 7 days and with persistent neutropenia was considered to belong to the same FN episode, as long as intravenous chemotherapy had not been restarted. Correspondingly, all outcomes were tracked within this 7-day period. ${ }^{8}$

The four main outcomes were FN, FN with bacteremia, with a serious medical complication (SMC), or with an SRE. Bacteremia was defined by detection of a recognized pathogen from one or more blood cultures according to current definitions. ${ }^{11,13}$ An SMC was defined as death due to any cause during $\mathrm{FN}$, admission to an intensive care unit, high dependency unit or other critical care unit for organ support, or severe sepsis (including septic shock) according to established definitions. ${ }^{14}$ SRE was defined as bacteremia or SMC. Infections other than bacteremia were thus included in the SRE definition if they led to an SMC.

\subsection{Statistical analysis}

Associations of the following 11 clinical characteristics with the four main outcomes were analyzed: sex, age group at screening, type of malignancy, relapse status, bone marrow involvement, chemotherapy intensity, presence of any central venous access device (CVAD), time since diagnosis, prior episodes of $\mathrm{FN}$, prior episodes of $\mathrm{FN}$ with bacteremia, and season of FN.

Chemotherapy intensity was split into four categories, defined according to the expected duration of severe neutropenia. ${ }^{15,16}$ Time since diagnosis was split into three categories, resulting from collapsing 13 initial categories by the adjacent categories method, applying threelevel mixed Poisson regression on FN with SRE. ${ }^{17,18}$ In Switzerland, viral infections are much more common during autumn and winter. Season was therefore split into two predefined categories, spring/summer (March to August) and autumn/winter (September to February). Port- a-caths were the most common reported CVAD systems, used in 160 (98\%) of 164 years of cumulative chemotherapy time. Therefore no further splitting of CVAD was done for analysis.

Analysis included univariate and multivariate three-level mixed Poisson regression analysis with chemotherapy time as rate multiplier. Random intercepts per patient, nested within center, were used to account for multiple randomization periods and $\mathrm{FN}$ episodes per patient. Interaction terms of clinical characteristics with the randomized fever limit were nonsignificant in all univariate analyses. Thus, confounding of the risk prediction analyses presented here by the randomized study design could be excluded.

Characteristics significantly associated in univariate analyses were used for the construction of multivariate mixed Poisson risk-prediction models (stepwise procedure, $p<.05$ for entry and for removal). ${ }^{19}$

A risk-prediction score was derived from the multivariate mixed Poisson model for FN with SRE. Its weights equaled the model coefficients multiplied by 2 and then rounded to the nearest integer. The score was the sum of these weights.

The predictive performance of this score was assessed by two measures. First, the area under the curve (AUC) of sensitivity versus time declared to be at risk was calculated as a global performance measure. Second, the time needed to cover (TNC) one event was calculated for all potential thresholds of the score, by dividing the time at risk by the number of events observed during this time. Time at risk was the cumulative chemotherapy time at or above a specific score threshold. Comparable to the number needed to treat (NNT) as an efficacy measure for treatments, the TNC is a measure for the potential efficacy of primary antibiotic prophylaxis. The TNC is directly linked to the time needed to prevent (TNP) one event with continuous antibiotic prophylaxis by the equation TNPc $=$ TNC/efficacy of prophylaxis. With perfect efficacy, TNPc equals TNC, but with nonperfect prophylaxis, TNPc exceeds TNC. Clinically, antibiotic prophylaxis is usually applied intermittently by, for example, delayed starting and stopping after resolution of severe neutropenia. ${ }^{7}$ The resulting clinically meaningful TNPi is the time needed to prevent one event by intermittent prophylaxis. Defined by TNPi $=$ TNPc*PTi, it is shorter than TNPc, because PTi, the proportion of time with intermittent prophylaxis, is below 1 by definition. Taken together, TNPi depends on the TNC described here, the efficacy of prophylaxis, and the proportion of time with intermittent prophylaxis as follows: TNPi $=(\mathrm{TNC} / \text { efficacy })^{*} \mathrm{PTi}$. To calculate TNPi, external information on efficacy and timing of intermittent antibiotic prophylaxis were extracted from a recently published randomized controlled trial. ${ }^{7}$

Multiple random replications of 10 -fold internal patient-level crossvalidation were used for three aims. First, 100 replications per model aimed to detect overfitting, defined by decreasing median AUC in successive steps, in stepwise multivariate model generation. Second, 100 replications per score aimed to confirm that increasing score complexity led to significantly increased score performance assessed by AUC. Third, 1000 replications of the final score aimed to give robust estimates of the score's TNCs at different thresholds.

The predictive performance of the risk-prediction score developed here was compared with the performance of published scores or clinical decision rules. Specifically, TNCs of the score in these 1000 
replications were compared with TNCs of published rules in 1000 patient-level bootstrapping replications of the dataset at comparable (ratio 0.9-1.1) proportions of time at risk.

Tests were two-sided throughout, $p$-values $<.05$ were considered significant and correspondingly, 95\% confidence intervals were calculated. All analyses were performed using R 3.6.3. ${ }^{20}$ Specifically, the "gImer" function from the "Ime4" library ${ }^{21}$ was used for mixed Poisson regression, the "gImmPQL" function from the "MASS" library ${ }^{22}$ when "glmer" failed, and the "pROC" library ${ }^{23}$ for AUC calculation.

\section{3 | RESULTS}

\subsection{Centers, patients, and FN episodes}

In one of the six participating SPOG centers recruiting patients for this study, systematic primary antibiotic prophylaxis was given to patients with acute myeloid leukemia (AML) and with high-risk or relapsed acute lymphoblastic leukemia (ALL). Results of the 238 patients recruited in the remaining five centers are analyzed here. Their median age at screening was 7 years (interquartile range, 412), and 97 (41\%) were female. Characteristics did not differ between patients studied and patients without IC on file, except for recruitment differences between centers (Table S1).

During 173 years (63,122 days) of cumulative chemotherapy time, $318 \mathrm{FN}$ episodes were recorded, 53 (17\%) of them with bacteremia, 28 (9\%) with SMC, and 68 (21\%) with at least one SRE (Table S2, Figure S1).

\subsection{Associations of clinical characteristics with FN episodes}

Nine of the 11 clinical characteristics studied were significantly associated with the risk to develop FN during chemotherapy in univariate analysis. Five of these characteristics remained significantly and independently associated with the risk to develop FN in multivariate analysis. These were chemotherapy intensity, time since diagnosis, prior episodes of FN, season and type of malignancy. Internal crossvalidation detected overfitting by inclusion of the last two characteristics (Tables S3 and S4).

Five clinical characteristics were significantly associated with FN with bacteremia in univariate analysis. Three remained significantly and independently associated with $\mathrm{FN}$ with bacteremia in multivariate analysis. These were chemotherapy intensity, time since diagnosis, and type of malignancy. Internal cross-validation detected overfitting by inclusion of the last characteristic (Tables S3 and S4).

Three clinical characteristics were significantly associated with FN with SMC in univariate analysis. Two remained significantly and independently associated with FN with SMC in multivariate analysis. These were chemotherapy intensity and type of malignancy. Internal crossvalidation detected no overfitting (Tables S3 and S4).

Six clinical characteristics were significantly associated with $\mathrm{FN}$ with SRE in univariate analysis (Table 1). Three remained significantly and independently associated with FN with SRE in multivariate analysis. These were, like for FN with bacteremia, chemotherapy intensity, time since diagnosis, and type of malignancy (Table 2). Internal crossvalidation detected no overfitting.

\section{3 | Scoring system to predict FN episodes with SRE}

The score predicting the risk to develop FN with SRE during chemotherapy was based on the multivariate model. Risk prediction, assessed by AUC, improved significantly in scores based on one versus two versus three variables (median of 100 cross-validations, 0.745 versus 0.768 versus $0.777 ; p<.001$ for both comparisons). The final risk-prediction score was thus based on three variables, chemotherapy intensity, time since diagnosis, and type of malignancy (Table 2). It had 15 levels, ranging from 0 to 14 (Figure 1, Table 3; Table S5 and S6). The noncross-validated AUC of this SRE-based score was 0.753 for prediction of $\mathrm{FN}$ as such, 0.840 for $\mathrm{FN}$ with bacteremia, 0.798 for $\mathrm{FN}$ with SMC, and 0.823 for FN with SRE.

\subsection{Performance of the score}

As expected, median cross-validated TNCs were slightly higher than noncross-validated TNCs for prediction of FN with SRE, bacteremia or SMC, and of FN as such (Figure 1; Figure S2, Table S7). For prediction of FN with SRE, two clinically useful score thresholds were found by graphical TNC analysis (Figure 1).

Applying the first threshold of scores $\geq 11$, including $2.3 \%$ chemotherapy time at risk, the TNC was 3.2 months per FN with SRE (median of cross-validations 4.1; Table 4). Clinically, this threshold included intensive chemotherapy cycles for patients with $A M L$ and mature B-cell non-Hodgkin lymphoma (NHL), and myeloablative therapy before autologous HSCT for patients with NHL and solid tumors outside the central nervous system (CNS), all during the first 7 months of chemotherapy (Table 3; Table S5 and S6).

Applying the second threshold of scores $\geq 8$, including $24.9 \%$ chemotherapy time at risk, the TNC was 9.9 (median of crossvalidations 12.1; Table 4). Clinically, this threshold additionally included intensive chemotherapy cycles for patients with ALL within the first 7 months of chemotherapy, and myeloablative therapy before autologous HSCT for patients with Hodgkin lymphoma, NHL, CNS tumors, and solid tumors outside the CNS irrespective of timing. These two thresholds were confirmed for prediction of FN with bacteremia, with SMC, and for FN as such (Figure S3, Table S7).

\subsection{External validation and performance of published models, scores, and rules}

A PubMed search for published risk-prediction models, scores, and rules up to February 9, 2021 using search terms for "fever," 
TA B LE 1 Associations of clinical characteristics with FN with SRE, univariate analysis

\begin{tabular}{|c|c|c|c|c|}
\hline \multirow[b]{2}{*}{ Characteristics } & \multicolumn{4}{|c|}{ FN with SRE } \\
\hline & CT & Events & Rate ratio $(95 \% \mathrm{CI})$ & $p$-Value \\
\hline Total & 173 & 68 & & \\
\hline \multicolumn{5}{|l|}{ Sex } \\
\hline Male & 96 & 41 & Reference & \\
\hline \multicolumn{5}{|c|}{ Age at screening (years) } \\
\hline $1-4$ & 54 & 30 & Reference & - \\
\hline $5-8$ & 52 & 16 & $0.57(0.31-1.04)$ & .067 \\
\hline $9-12$ & 29 & 6 & $0.38(0.16-0.92)$ & .032 \\
\hline
\end{tabular}

Disease-related characteristics

Type of malignancy

Acute lymphoblastic leukemia

Acute myeloid leukemia

Hodgkin lymphoma

Non-Hodgkin lymphoma

Central nervous system tumor

Other solid tumor

Relapse status

No relapse

Relapsed malignancy

13

$\begin{array}{ll}105 & 41 \\ 4 & 10 \\ 7 & 1 \\ 10 & 6 \\ 17 & 2 \\ 31 & 8\end{array}$

41

10

1

8

63

5

Bone marrow involvement

None

Bone marrow involvement

168

5

Characteristics of therapy and course

Chemotherapy intensity (expected neutropenia)

1 (none)
2 ( $\leq 10$ days)
3 (>10 days)
4 (myeloablative)

Central venous access device

None

Any type implanted

$80 \quad 5$

$88 \quad 46$

$3-14$

13

Time since diagnosis

$<1$ month

1 to $<8$ months

$\geq 8$ months

90

$164 \quad 68$

Prior episode of FN

No

Yes

9

74

90

12

47

9

10

5

46

14

3

0

68

Prior episode of $\mathrm{FN}$ with bacteremia ${ }^{\mathrm{a}}$

$\begin{array}{llllll}\text { No } & 142 & 52 & \text { Reference } & - & \\ \text { Yes } & 31 & 16 & 1.29(0.68-2.45) & .43 & \text { (Continues) }\end{array}$

Reference

$7.45(2.81-19.8) \quad<.001$

$0.35(0.05-2.70) \quad .32$

$1.67(0.61-4.56) \quad .32$

$0.29(0.07-1.28) \quad .10$

$0.67(0.30-1.51) \quad .33$

Reference

$1.36(0.42-4.39)$

.61

Reference

$4.97(2.36-10.5)$

$<.001$

Reference

$8.73(3.43-22.2) \quad<.001$

$72.9(23.7-224)<.001$

$29.3(6.45-133)<<.001$

Reference

Model failure

$14.2(5.80-35.0)$

$<.001$

$6.30(3.01-13.2)$

$<.001$

Reference

$-$

Reference

$0.75(0.41-1.38)$

.36

(Continues) 
TABLE 1 (Continued)

\begin{tabular}{|c|c|c|c|c|}
\hline \multirow[b]{2}{*}{ Characteristics } & \multicolumn{4}{|c|}{ FN with SRE } \\
\hline & $\overline{C T}$ & Events & Rate ratio $(95 \% \mathrm{Cl})$ & $p$-Value \\
\hline \multicolumn{5}{|l|}{ Season } \\
\hline Spring/Summer & 99 & 30 & Reference & - \\
\hline Autumn/Winter & 74 & 38 & $1.86(1.13-3.06)$ & .015 \\
\hline
\end{tabular}

Note: Displayed are results of univariate three-level mixed Poisson regression.

Abbreviations: $\mathrm{Cl}$, confidence interval; $\mathrm{CT}$, chemotherapy time in years; $\mathrm{FN}$, fever in neutropenia; SRE, safety-relevant event.

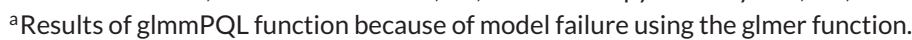

"neutropenia," and "children," including forward citation searching, gave two results. Wicki et al. reported in 2008 on a retrospective single-center study. ${ }^{24}$ Higher chemotherapy intensity, shorter time since diagnosis, prior episodes of $\mathrm{FN}$, bone marrow involvement, and presence of a CVAD were significantly associated with the risk to develop FN and FN with bacteremia. Applied on the dataset described here, the Wicki multivariate model resulted in 260 levels. For 6 of 7 score thresholds $\geq 8$, comparisons of TNCs with corresponding Wicki thresholds were possible. The cross-validated TNCs for SRE of the score developed here were always comparable (within 1 day; thresholds $8,9,10,14$ ) or significantly lower (thresholds 12,13 ) than the corresponding median bootstrapped TNC of Wicki et al. (Figure 1, Table 4).

A guideline published by Lehrnbecher et al. in 2020 recommends antibacterial prophylaxis for pediatric patients with $\mathrm{AML}$ and relapsed ALL during intensive chemotherapy. ${ }^{5}$ At the corresponding threshold $\geq 11$ (time at risk, $2.3 \%$ vs. $3.1 \%$ ), the median cross-validated TNC for SRE of the score developed here was significantly lower than the median bootstrapped Lehrnbecher TNC $(p<.001$; Table 4, Figure 1).

Using external information on efficacy and timing of intermittent prophylaxis from a recently published randomized controlled trial, ${ }^{7}$ the TNPi was 4.3 months per FN with bacteremia and 5.2 months per FN with SRE using a threshold of $\geq 11$ (Table 5).

\section{DISCUSSION}

This analysis developed a score predicting the risk for FN with SRE in pediatric patients treated with chemotherapy for cancer. At least one SRE was reported in $21 \%$ of FN episodes. The score developed here was based on three easily accessible clinical characteristics, intensity of chemotherapy, time since diagnosis, and type of malignancy.

The overall performance of this score to predict FN with SRE was good, the median cross-validated AUC of sensitivity versus proportion of time at risk was 0.777 (noncross-validated 0.823 ).

However, the predictive performance in high-risk situations is more important than the overall performance for targeted primary antibiotic prophylaxis. The TNC introduced here is a quantitative measure of the maximum effect size of such primary prophylaxis. It can help to weigh potential benefits versus adverse events and further drawbacks of prophylaxis. A restrictive score threshold of $\geq 11$ resulted in $2.3 \%$ high-risk time, with a cross-validated TNC one FN with SRE of 4.1 months, which corresponds to 5.2 months of intermittent prophylaxis to prevent one FN with SRE (TNPi).

The score and its TNC/TNPi results can be mainly used for three purposes. First, if the prevention of one FN episode with SRE is considered to outweigh the drawbacks of, for example, half a year of antibiotic prophylaxis, a threshold of $\geq 11$ can directly serve as a clinical decision rule to define patient groups for targeted primary antibiotic prophylaxis. Second, for patient groups with intermediate $\mathrm{TNCi}$, for example, from 6 to 12 months, the results can inform power analysis and further design questions of future efficacy studies of antibiotic prophylaxis. Third, efficacy studies should be avoided in patient groups with TNPi above a clinically meaningful limit of,for example, 12 months.

Four randomized, placebo-controlled studies have analyzed the efficacy of antibacterial prophylaxis in children undergoing chemotherapy for hematologic malignancies or solid tumors. ${ }^{725-27}$ Results ranged from no effect ${ }^{25}$ to an efficacy of $0.31^{26}$ for the reduction of $\mathrm{FN}$. Only one study, investigating the effect of levofloxacin, had enough power to investigate efficacy of prophylaxis in reducing FN with bacteremia (0.48) and severe infections (0.38). ${ }^{7}$ Meta-analyses in adult and pediatric patients had found prophylaxis with fluoroquinolones to be efficacious in certain patient groups, but report inconsistent data on resistance. ${ }^{28}$ A future UK study will compare ciprofloxacin prophylaxis versus standard of care during induction therapy in pediatric patients with ALL treated in the ALLTogether-1 study. ${ }^{29}$

For the prediction of FN with SRE, the score developed here significantly outperformed a more complex published prediction model based on five variables ${ }^{24}$ and a recent clinical decision rule on antibiotic prophylaxis. ${ }^{5}$ The FENCE score, ${ }^{30}$ developed in adult patients to assess the FN risk during the first cycle of chemotherapy, confirmed the relevance of two of the three score components, type of malignancy and chemotherapy intensity, irrespective of patient age.

A limitation of this analysis is that information on other comorbidities and biochemical markers that might influence the risks analyzed was not available. A second limitation is the small number of patients treated with intensive chemotherapy, such as AML-type and myeloablative regimens. ${ }^{8}$ Before clinical application in these patients, this score should be validated prospectively. A third limitation is that the centers used different antibiotics for treatment of FN. Validation studies have to show, how this impacts the generalizability of the results. A fourth limitation is the single-country setting of the study, Switzerland being a high-income country. These results are thus only valid for countries with comparable settings. 


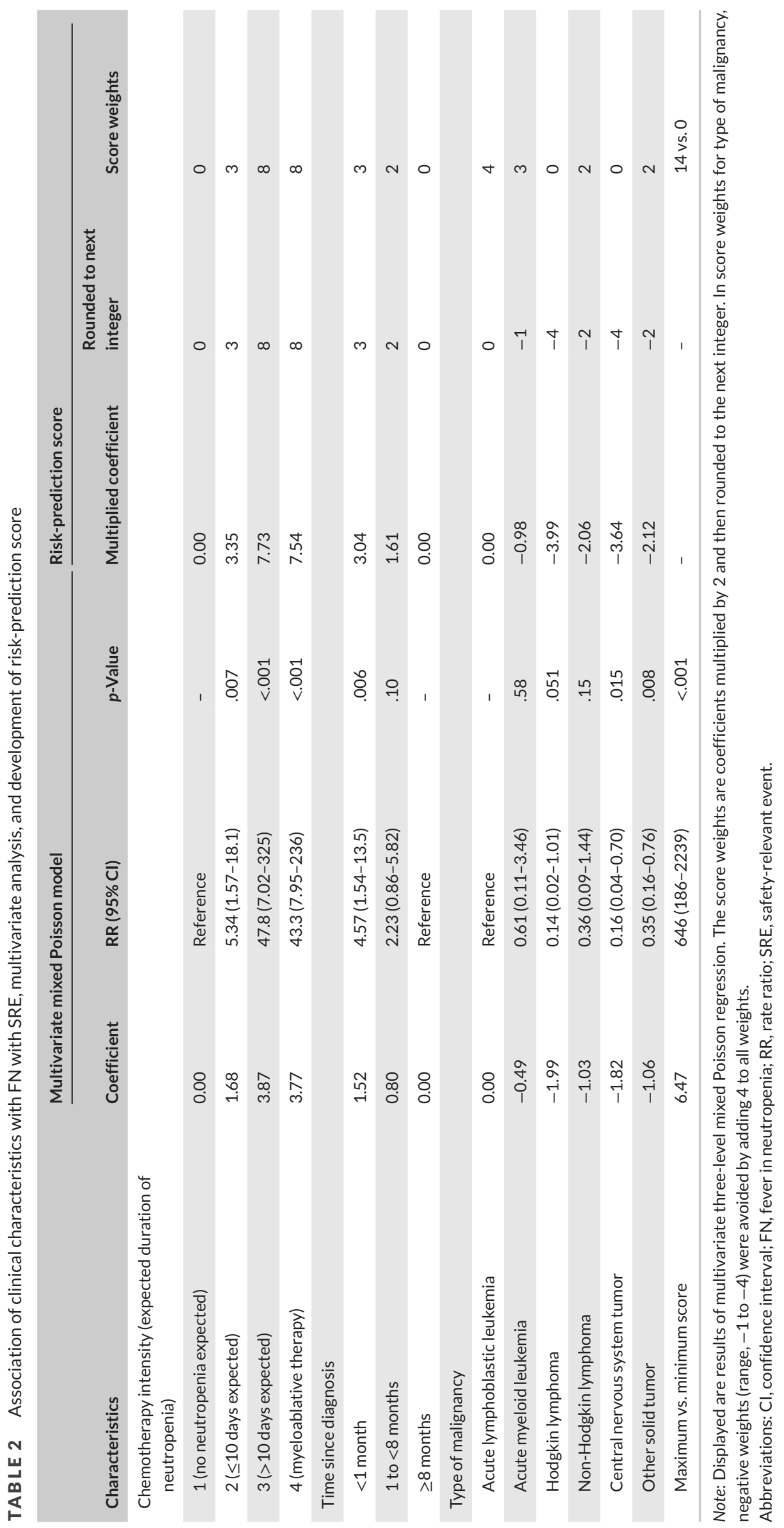




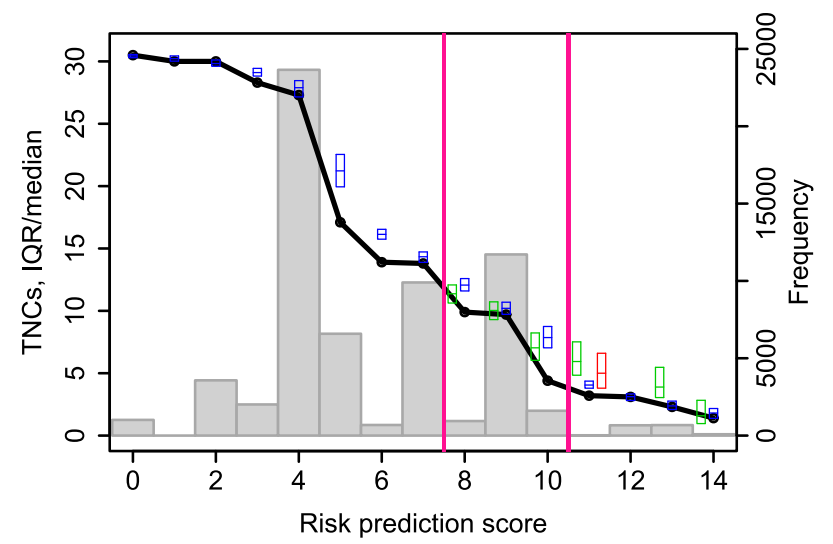

FIGURE 1 Frequencies of risk-prediction scores in the FN episodes studied here, plus the corresponding TNCs. The histogram, displayed in grey, shows the frequency distribution of the risk-prediction score developed here (SPOG 2015 FN Definition score; $y$-axis on the right). The remaining information is on the corresponding time needed to cover (TNC) using different score thresholds ( $y$-axis on the left). Specifically, open circles joined by a solid black line indicate the noncross-validated TNCs of the score. The boxplots show median and interquartile range (IQR) of cross-validated TNCs (blue), of bootstrapped TNCs for corresponding thresholds from Wicki et al. (green), and from Lehrnbecher et al. (red)

Major strengths of this analysis are its large base of prospectively collected data in a multicenter study setting with nonbiased recruitment of patients with a wide and representative spectrum of malignan- cies. Internal cross-validation avoided overfitting of the multivariate model and, thus, the score. Nonsignificant results of interaction analyses excluded relevant confounding of the score by the randomized design of the underlying trial. The score developed here is easy to calculate based on three routinely accessible patient characteristics and is applicable during the entire chemotherapy.

In conclusion, the score developed here, based on three routinely accessible characteristics, accurately identifies pediatric patients with cancer at risk to develop FN with SRE during chemotherapy. The score, together with the effect size measures presented, can help to design clinical decision rules on targeted primary antibiotic prophylaxis and corresponding efficacy studies.

\section{ACKNOWLEDGMENTS}

We thank all patients and parents for trial participation; Cécile Adam, MD, Nannette Keller, MSc, Kurt Leibundgut, MD, David Nadal, MD, Felix Niggli, MD, Martina Peluso, MSc, Arne Simon, MD, Oliver Teuffel, MD, Karin Zimmermann, PhD, and Nicolas $X$ von der Weid, MD for help in protocol development and in the Study Committee; Johannes Rischewski, MD for support in the Study Committee; Renate Siegenthaler, Friedgard Julmy, Rodolfo Lo Piccolo, Sarah Blanc, Janine Garibay, and Beate Schwencke for data management; and Daniel Ammann for database development and maintenance. This trial was supported by an unrestricted research grant from the Swiss Cancer League (KLS-3645-02-2015) and the Berner Stiftung für krebskranke Kinder.

TAB LE 3 Scores of common clinical situations, sorted according to scores, 7-14

\begin{tabular}{|c|c|c|c|}
\hline Score & Malignancy and therapy & Chemotherapy intensity & $\begin{array}{l}\text { Time since } \\
\text { diagnosis(months) }\end{array}$ \\
\hline $14=3+8+3$ & AML intensive & 3 & $<1$ \\
\hline $13=2+8+3$ & Mature B-NHL & 3 & $<1$ \\
\hline $13=3+8+2$ & AML intensive & 3 & 1 to $<8$ \\
\hline $12=2+8+2$ & Mature B-NHL & 3 & 1 to $<8$ \\
\hline $12=2+8+2$ & NHL auto HSCT & 4 & 1 to $<8$ \\
\hline $12=2+8+2$ & Solid auto HSCT & 4 & 1 to $<8$ \\
\hline $10=4+3+3$ & ALL intensive & 2 & $<1$ \\
\hline $10=0+8+2$ & $\mathrm{HL}$ auto HSCT & 4 & 1 to $<8$ \\
\hline $10=2+8+0$ & Solid auto HSCT & 4 & $\geq 8$ \\
\hline $9=4+3+2$ & ALL intensive & 2 & 1 to $<8$ \\
\hline $8=0+8+0$ & HL auto HSCT & 4 & $\geq 8$ \\
\hline $8=2+3+3$ & NHL intensive & 2 & $<1$ \\
\hline $8=0+8+0$ & CNS auto HSCT & 4 & $\geq 8$ \\
\hline $8=2+3+3$ & Solid intensive & 2 & $<1$ \\
\hline
\end{tabular}

Abbreviations: ALL, acute lymphoblastic leukemia; AML, acute myeloid leukemia; auto, autologous; B-NHL, B-cell non-Hodgkin lymphoma; CNS, central nervous system; HL, Hodgkin lymphoma; HSCT, hematopoietic stem cell transplantation; NHL, non-Hodgkin lymphoma. 


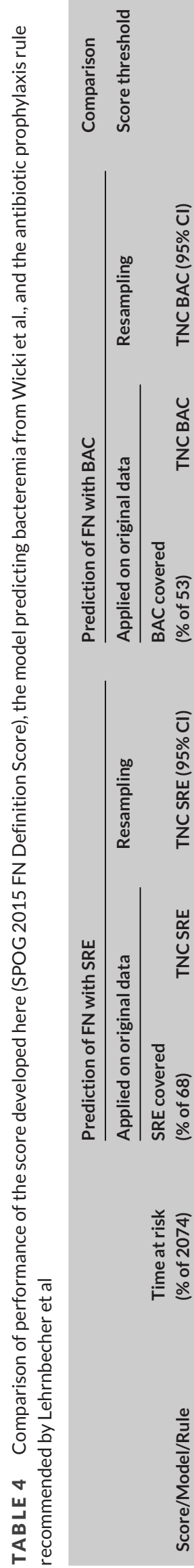

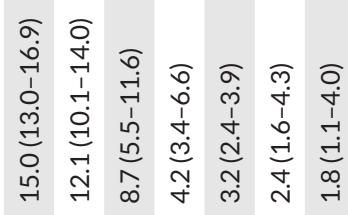

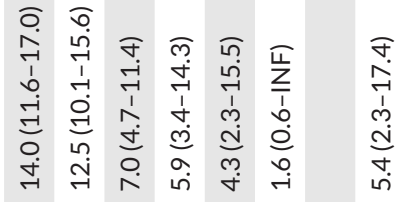

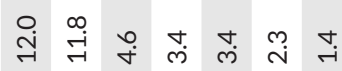

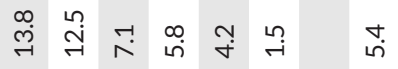

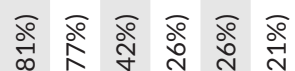

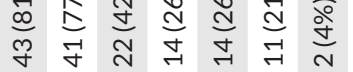

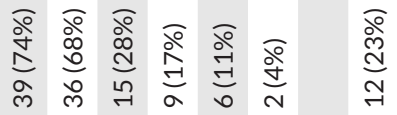<smiles>C1CCCC1</smiles>

बें

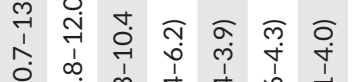

गुำ

每

每

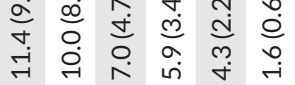

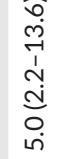

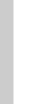

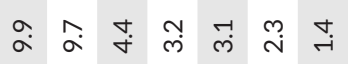

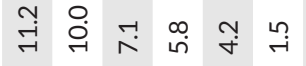

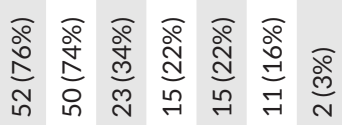

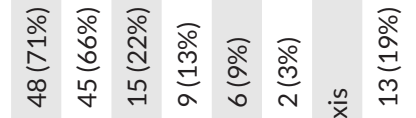

\section{wer}

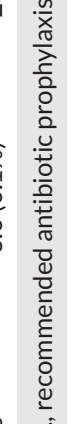

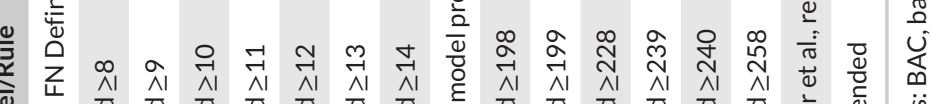

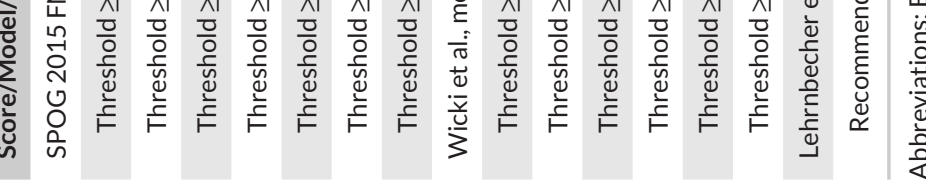




\section{CONFLICT OF INTEREST}

Jochen Roessler reports personal fees from SOBI, Roche and Pierre fabre, all for Advisory Board membership, outside the submitted work. The remaining authors declare no conflict of interest.

\section{DATA AVAILABILITY STATEMENT}

The study protocol, including the predefined prediction part presented here, is available on https://www.spog.ch/wp-content/uploads/2020/ 03/SPOG_FN_Protocol_1.1_20161123_PDF.pdf. Participant data are available for qualified researchers who wish to access the data following article publication; no end data. Proposals should be directed to roland.ammann@insel.ch; to gain access, data requestors will need to sign a data access agreement.

\section{ORCID}

Christa Koenig (D) https://orcid.org/0000-0002-9965-5183

Jochen Roessler (D) https://orcid.org/0000-0003-4022-4917

Roland A. Ammann (D) https://orcid.org/0000-0002-1902-2352

\section{REFERENCES}

1. Bodey GP, Buckley M, Sathe YS, Freireich EJ. Quantitative relationships between circulating leukocytes and infection in patients with acute leukemia. Ann Intern Med. 1966;64:328-340.

2. Lehrnbecher T, Robinson P, Fisher B, et al. Guideline for the management of fever and neutropenia in children with cancer and hematopoietic stem-cell transplantation recipients: 2017 update. J Clin Oncol. 2017;35:2082-2094.

3. Bochennek K, Luckowitsch M, Lehrnbecher T. Recent advances and future directions in the management of the immunocompromised host. Semin Oncol. 2020;47:40-47.

4. Egan G, Robinson PD, Martinez JPD, et al. Efficacy of antibiotic prophylaxis in patients with cancer and hematopoietic stem cell transplantation recipients: a systematic review of randomized trials. Cancer Med. 2019;8:4536-4546.

5. Lehrnbecher T, Fisher BT, Phillips B, et al. Guideline for antibacterial prophylaxis administration in pediatric cancer and hematopoietic stem cell transplantation. Clin Infect Dis. 2019;71:226-236.

6. Lehrnbecher T, Averbuch D, Castagnola E, et al. 8th European Conference on Infections in Leukaemia: 2020 guidelines for the use of antibiotics in paediatric patients with cancer or post-haematopoietic cell transplantation. Lancet Oncol. 2021;22(6):e270-e280.

7. Alexander S, Fisher BT, Gaur AH, et al. Effect of levofloxacin prophylaxis on bacteremia in children with acute leukemia or undergoing hematopoietic stem cell transplantation: a randomized clinical trial. JAMA. 2018;320:995-1004.

8. Koenig C, Bodmer N, Agyeman PKA, et al. $39.0^{\circ} \mathrm{C}$ versus $38.5^{\circ} \mathrm{C}$ ear temperature as fever limit in children with neutropenia undergoing chemotherapy for cancer: a multicentre, cluster-randomised, multiple-crossover, non-inferiority trial. Lancet Child Adolesc Health. 2020;4:495-502.

9. Harris PA, Taylor R, Thielke R, Payne J, Gonzalez N, Conde JG. Research electronic data capture (REDCap)-a metadata-driven methodology and workflow process for providing translational research informatics support. J Biomed Inform. 2009;42:377-381.

10. SPOG 2015 FN Definition Protocol. Swiss Paediatric Oncology Group. 2016. Accessed May 11, 2021. https://www.spog.ch/wpcontent/ uploads/2020/03/SPOG_FN_Protocol1.1_20161123_PDF.pdf

11. Haeusler GM, Phillips RS, Lehrnbecher T, Thursky KA, Sung L, Ammann RA. Core outcomes and definitions for pediatric fever and neutropenia 
research: a consensus statement from an international panel. Pediatr Blood Cancer. 2015;62:483-489.

12. Freifeld AG, Bow EJ, Sepkowitz KA, et al. Clinical practice guideline for the use of antimicrobial agents in neutropenic patients with cancer: 2010 update by the infectious diseases society of America. Clin Infect Dis. 2011;52:e56-e93.

13. Centre for Disease Control and Prevention (CDC) Central lineassociated bloodstream infection (CLABSI) event. Accessed May 11, 2021. https://www.cdc.gov/nhsn/PDFs/pscManual/4PSC_CLABS current.pdf

14. Goldstein B, Giroir B, Randolph A. International pediatric sepsis consensus conference: definitions for sepsis and organ dysfunction in pediatrics. Pediatr Crit Care Med. 2005;6:2-8.

15. Kern WV, Cometta A, De Bock R, Langenaeken J, Paesmans M, Gaya $\mathrm{H}$. Oral versus intravenous empirical antimicrobial therapy for fever in patients with granulocytopenia who are receiving cancer chemotherapy. International Antimicrobial Therapy Cooperative Group of the European Organization for Research and Treatment of Cancer. N Engl J Med. 1999;341:312-318.

16. Schlapbach LJ, Aebi C, Otth M, Luethy AR, Hirt A, Ammann RA. Serum levels of mannose-binding lectin and the risk of fever in neutropenia pediatric cancer patients. Pediatr Blood Cancer. 2007;49:11-16.

17. Byar DP. Identification of prognostic factors. In: Buyse ME, Staquet MJ, Sylvester RJ, eds. Cancer Clinical Trial Methods in Practice. Oxford Medical Publications; 1988:423-443.

18. Sallenbach S, Thiel S, Aebi C, et al. Serum concentrations of lectinpathway components in healthy neonates, children and adults: mannan-binding lectin (MBL), $\mathrm{M}-, \mathrm{L}-$, and $\mathrm{H}$-ficolin, and $\mathrm{MBL}-$ associated serine protease-2 (MASP-2). Pediatr Allergy Immunol. 2011;22:424-430

19. Altman D. Practical Statistics for Medical Research. Chapman \& Hall; 1991.

20. R Core Team. R: a Language and Environment for Statistical Computing. $R$ Foundation for Statistical Computing; 2021.

21. Bates D, Mächler M, Bolker B, Walker S. Fitting linear mixed-effects models using Ime4. J Stat Softw. 2015;67:48.

22. Venables WN, Ripley BD. Modern Applied Statistics with S. 4th ed. Springer; 2002.

23. Robin X, Turck N, Hainard A, et al. pROC: an open-source package for $\mathrm{R}$ and $\mathrm{S}+$ to analyze and compare ROC curves. BMC Bioinformatics. 2011;12:77.
24. Wicki S, Keisker A, Aebi C, Leibundgut K, Hirt A, Ammann RA. Risk prediction of fever in neutropenia in children with cancer: a step towards individually tailored supportive therapy? Pediatr Blood Cancer. 2008;51:778-783.

25. Widjajanto PH, Sumadiono S, Cloos J, Purwanto I, Sutaryo S, Veerman AJ. Randomized double blind trial of ciprofloxacin prophylaxis during induction treatment in childhood acute lymphoblastic leukemia in the WK-ALL protocol in Indonesia. J Blood Med. 2013;4:1-9.

26. Castagnola E, Boni L, Giacchino M, et al. A multicenter, randomized, double blind placebo-controlled trial of amoxicillin/clavulanate for the prophylaxis of fever and infection in neutropenic children with cancer Pediatr Infect Dis J. 2003;22:359-365.

27. Laoprasopwattana K, Khwanna T, Suwankeeree P, Sujjanunt T, Tunyapanit W, Chelae S. Ciprofloxacin reduces occurrence of fever in children with acute leukemia who develop neutropenia during chemotherapy. Pediatr Infect Dis J. 2013;32:e94-e98.

28. Gafter-Gvili A, Fraser A, Paul M, et al. Antibiotic prophylaxis for bacterial infections in afebrile neutropenic patients following chemotherapy. Cochrane Database Syst Rev. 2012;1(1):CD004386.

29. CiproPAL (ciprofloxacin prophylaxis in acute leukaemia) (CiproPAL). Identifier NCT04678869. https://www.clinicaltrials.gov/ct2/show/ NCT04678869. Accessed May 11, 2021.

30. Aagaard T, Roen A, Reekie J, et al. Development and validation of a risk score for febrile neutropenia after chemotherapy in patients with cancer: the FENCE score. JNCI Cancer Spectr. 2018;2(4):pky053.

\section{SUPPORTING INFORMATION}

Additional supporting information may be found online in the Supporting Information section at the end of the article.

How to cite this article: Lavieri L, Koenig C, Bodmer N, et al. Predicting fever in neutropenia with safety-relevant events in children undergoing chemotherapy for cancer: The prospective multicenter SPOG 2015 FN Definition Study. Pediatr Blood Cancer. 2021;68:e29253.

https://doi.org/10.1002/pbc.29253 